\title{
Characterization and Quantification of Biosynthesized Gold Nanoparticles Using Chenopodium aristatum $L$. Stem Extract
}

\author{
Can Huo ${ }^{1}$ Chun-Gang Yuan ${ }^{1} \cdot$ Yu-Kai Li ${ }^{1}$. \\ Peng-Le Liu ${ }^{1} \cdot$ Jing-Fu Liu ${ }^{2}$
}

Received: 14 May 2017/Published online: 17 July 2017

(C) Springer Science+Business Media, LLC 2017

\begin{abstract}
A cost-effective, and rapid method to synthesize gold nanoparticles using Chenopodium aristatum L. stem extract was developed in this study. Chenopodium aristatum $L$. is an obnoxious weed. Their tenacious vitality can provide us enormous biomass for beneficial utilizations. In this paper, its stem extract was used as both capping and reducing agents for the biosynthesis of gold nanoparticles (AuNPs). UV-Vis spectra showed surface plasmon resonance (SPR) band at $\sim 540 \mathrm{~nm}$. The synthesized AuNPs were quantified by the centrifugal ultrafilter method followed with ICP-MS determination. TEM images showed that the shapes of synthesized AuNPs were predominantly spherical. In addition, truncated triangular and small amounts of other shaped NPs were also formed. The results of SAED showed clear circular spots. The face-centered cubic structure of the AuNPs was confirmed by XRD peaks at $38.188^{\circ}, 44.552^{\circ}, 64.589^{\circ}$, and $77.584^{\circ}$, which corresponded to (111),

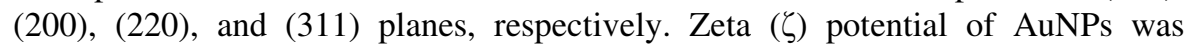
$-22.9 \pm 5.56 \mathrm{mV}$. FTIR spectrum showed the functional groups, such like $\mathrm{O}-\mathrm{H}$, $\mathrm{N}-\mathrm{H}, \mathrm{C}-\mathrm{H}, \mathrm{C}=\mathrm{O}$, were possibly responsible for reducing $\mathrm{Au}(\mathrm{III})$ ions and stabilizing nanoparticles. The synthesized AuNPs exhibited good catalytic and antioxidant activities in degradation of 4-nitrophenol and free radical scavenging.
\end{abstract}

Keywords Gold nanoparticles · Biosynthesis · Catalytic activity · Antioxidant activity

Chun-Gang Yuan

chungangyuan@hotmail.com

1 Department of Environmental Science and Engineering, North China Electric Power University, Baoding 071000, China

2 State Key Laboratory of Environmental Chemistry and Ecotoxicology, Research Center for Eco-Environmental Sciences, Chinese Academy of Sciences, Beijing 100085, China 


\section{Introduction}

Due to the widespread application of AuNPs in many fields like medicine [1], disease diagnostic [2], drug delivery systems [3], and fast-moving custom goods [4], there is an ever-growing need to develop a green method for the synthesis of AuNPs. However, the traditional chemical synthesis techniques are normally energy consuming or environment harmful/hazardous resulting from the use of complex and expensive equipment or toxic chemicals. Hence, biosynthesis is an alternative method to synthesize nanoparticles in a simple, low-cost and ecofriendly way. Both microorganisms and plants have the favorable abilities to synthesize AuNPs [5-8]. Plants have marvelous advantages over microorganisms for their rich biodiversity, cost effectiveness and industrial feasibility. In addition, the synthesis rates of plants extract are always faster than that of microorganisms $[9,10]$.

It was reported that the biosynthesized AuNPs exhibited marvelous catalytic/ photocatalytic activities of organic pollutants due to a high number of vacant reactive surface sites and high surface area [11]. Many hazardous and nonbiodegradable colored dyes or nitrophenols have been degraded in the presence of biosynthesized AuNPs [12-14]. Good antioxidant activities of biosynthesized AuNPs were also reported [15, 16]. These properties of biosynthesized AuNPs encourage us to explore more effective ways for their synthesis.

In this study, we used Chenopodium aristatum L. (C. aristatum L.) stem extract both as reducing and stabilizing agents for the synthesis of AuNPs. $C$. aristatum $L$. is one of the members of Chenopodiaceae family and considered as an obnoxious weed in the north of China. C. aristatum L. is rich of flavonoids (mainly kaempferol and quercetin glucosides), phenolic acids and terpenoids [17-19]. These compounds are able to reduce metal ions into metal nanoparticles $[20,21]$. Therefore, the stem extract of $C$. aristatum $L$. has the potential to effectively synthesize AuNPs. Considering the bioactive compounds in it and its availability, $C$. aristatum $L$. can provide us enormous biomass as a natural factory for the synthesis of nanoparticles. More importantly, to use weed for biosynthesis can provide a cheaper way compared with fruits, vegetables and ornamental plants [16, 22].

From an engineering prospective, it is important to investigate the yield of the biosynthesized NPs in the mixture. Many researchers studied the NPs yields only based on the absorbance of UV-Vis spectra. Little attention was devoted to exact quantification of the produced amounts of the NPs during the reactions. UV-Vis analysis can only give us some information about relative extent of reaction. It cann't provide us the exact transformation efficiency from ions to nanoparticles. In recent years, ICP-MS has been wildly used for the quantification of NPs, which supply us a powerful tool to monitor the real yield of biosynthesis procedure [23-25]. At present study, we determined the amounts of AuNPs prepared using the C. aristatum $L$. stem extract by ICP-MS quantification after centrifugal ultrafiltration. 
Herein, we developed a novel method to synthesize AuNPs using C. aristatum $L$. stem extract. The prepared AuNPs was well characterized, and the yield of the AuNPs was also quantified. In addition, the catalytic and antioxidant activities of AuNPs were investigated.

\section{Materials and Methods}

\section{Materials}

Chenopodium aristatum $L$. was collected in local roadside land and thoroughly washed by deionized water before use. The stem of $C$. aristatum $L$. was carefully cut with a knife and dried in the shade. The dried stems were ground to powder and sieved with 100 mesh sieve for the extract preparation. Chloroauric acid $\left(\mathrm{HAuCl}_{4} \cdot 4 \mathrm{H}_{2} \mathrm{O}\right)$ was procured from Sinopharm Chemical Reagent Co. Ltd. 4-nitrophenol, sodium borohydride and sodium hydroxide were purchased from Kermel Chemical Reagent (Tianjin, China). DPPH (1, 1-Diphenyl-2-Picryl hydroxyl) and ABTS (2,2'-Azinobis-(3-ethylbenzthiazoline-6-sulphonate)) were obtained from Sigma-aldrich (America). All glassware was washed by fresh aqua regia solution and deionized water thoroughly before the experiment.

\section{Synthesis of AuNPs}

The dried C. aristatum L. stem powder $(1.0 \mathrm{~g})$ was boiled in $100 \mathrm{~mL}$ of deionized water for $15 \mathrm{~min}$ and cooled to the room temperature. Later on, the extraction was filtered through $100 \mathrm{~nm}$ membrane, and the filtrate was used as reducing and stabilizing agent for the followed experiments. $50 \mu \mathrm{L}$ of $\mathrm{HAuCl}_{4} \cdot 4 \mathrm{H}_{2} \mathrm{O}(100 \mathrm{mM})$ was added into $9.95 \mathrm{~mL}$ of the prepared extract and vortexed thoroughly. The mixture was stood at room temperature for $24 \mathrm{~h}$. The obtained AuNPs were centrifuged and cleaned with deionized water for 3 times to remove the remained biomolecules. The pellet was re-dispersed in the deionized water and stored at $4{ }^{\circ} \mathrm{C}$ for the further studies.

The influence of the concentration of $\mathrm{HAuCl}_{4} \cdot 4 \mathrm{H}_{2} \mathrm{O}$ was studied by changing the added volumes of the $\mathrm{HAuCl}_{4} \cdot 4 \mathrm{H}_{2} \mathrm{O}$ solution. 25,50 and $100 \mu \mathrm{L}$ of $\mathrm{HAuCl}_{4} \cdot 4 \mathrm{H}_{2} \mathrm{O}$ $(100 \mathrm{mM})$ solution was respectively added into the stem extract to keep the final volume of the mixture at $10 \mathrm{~mL}$. The effect of reaction temperature on the synthesis of AuNPs was studied by adding $50 \mu \mathrm{L}$ of $\mathrm{HAuCl}_{4} \cdot 4 \mathrm{H}_{2} \mathrm{O}(100 \mathrm{mM})$ solution into $9.95 \mathrm{~mL}$ of the stem extract and incubating the mixture at the different temperatures $\left(40,50\right.$ and $60{ }^{\circ} \mathrm{C}$, respectively) in a water bath. In order to investigate the effect of extract $\mathrm{pH}$, the $\mathrm{pH}$ was adjusted to the specific value in the range of 2-10 using $0.1 \mathrm{M} \mathrm{NaOH}$ and $0.1 \mathrm{M} \mathrm{HCl}$ solution. $50 \mu \mathrm{L}$ of $\mathrm{HAuCl}_{4} \cdot 4 \mathrm{H}_{2} \mathrm{O}(100 \mathrm{mM})$ solution was added into $9.95 \mathrm{~mL}$ of the stem extract as the precursors of AuNPs for each 
experiment. All of the synthesis procedures were monitored by a UV-Vis spectrometer.

\section{Characterization of the Synthesized AuNPs}

The formation of AuNPs was monitored by a UV-Vis spectrometer (T6, Purkinje General, China) from 300 to $800 \mathrm{~nm}$ at a resolution of $1 \mathrm{~nm}$. All samples were diluted 6 times using deionized water. The deionized water was used as the blank. The yield of the AuNPs was measured by inductively coupled plasma-mass spectrometry (Agilent 7500ce, America). The synthesized AuNPs and remained $\mathrm{Au}(\mathrm{III})$ ions in the solution were separated by a ultrafiltration tube (Amicon Ultra$15,3 \mathrm{KD}$, Millipore, MA) at 10,000 rpm for $40 \mathrm{~min}$. Then, the concentration of gold in the filtrate was determined and represented the remained $\mathrm{Au}$ ions in the mixture. The reacted Au ions (AuNPs) were obtained by subtracting the remained $\mathrm{Au}$ ions in the filtrate from the total amount. The reaction yield was calculated by comparing the reacted $\mathrm{Au}$ ions (presented as AuNPs) with the total amount of $\mathrm{Au}$ ions in the original solution. The zeta potential was monitored by dynamic light scattering (DLS) with a Nano Zeta sizer (Malvern Instruments Ltd. Malvern, UK) at $25^{\circ} \mathrm{C}$. The size and morphology of the prepared AuNPs were characterized by transmission electron microscope (Tecnai G2 F20 S-TWIN, FEI/Netherland). Samples were prepared by dropping a drop of synthesized AuNPs colloid onto a carbon-coated $\mathrm{Cu}$ grid and evaporated in ambient condition. The microscope was also equipped with a genesis liquid nitrogen cooled energy-dispersive X-ray analysis (EDS) detector for detailed elemental analysis. The crystalline structure of synthesized AuNPs was studied by the X-ray powder diffractometer (D8 ADVENCE, Bruker/Switzerland) with $\mathrm{Cu}-\mathrm{K} \alpha$ radiation $(\lambda=1.5418 \AA)$ with a scan speed of $2 \% \mathrm{~min}$. Fourier transform infrared (Magna-IR 750/America) was carried out to find the functional groups which were possibly responsible for reducing the $\mathrm{Au}(\mathrm{III})$ ions to $\mathrm{Au}(0)$ and stabilizing the AuNPs without agglomeration. FTIR spectrophotometer was operated in the spectral range $4000-400 \mathrm{~cm}^{-1}$ with resolution of $4 \mathrm{~cm}^{-1}$.

\section{Catalytic Activity of the Synthesized AuNPs}

The catalytic activity of the synthesized AuNPs was studied by degradation of 4-nitrophenol (4-NP) to 4-aminophenol (4-AP) in the presence of $\mathrm{NaBH}_{4}$. The typical experimental procedure was carried out as follows: $200 \mu \mathrm{L}$ of freshly prepared $\mathrm{NaBH}_{4}(5 \mathrm{M})$ and $20 \mu \mathrm{L}$ of $4-\mathrm{NP}(20 \mathrm{mM})$ were added into a standard quartz cell and mixed with $2740 \mu \mathrm{L}$ of sterile deionized water. $40 \mu \mathrm{L}$ of the prepared AuNPs colloid was subsequently added into the above mixture as a catalyst. The various volumes of AuNPs colloid were added into the mixture to investigate the dose effect on the degradation efficiency. The degradation progress was monitored using a UV-Vis spectrometer at the specific time intervals. 


\section{Antioxidant Activity of AuNPs}

\section{DPPH Radical Scavenging Assay}

The DPPH radical scavenging activity of the AuNPs was analysed according to the method given by Kumar et al. [22, 26] with slight modification. Different concentrations of AuNPs $(75,150,225,300$, and $375 \mu \mathrm{M})$ were added into $0.1 \mathrm{mM}$ of methanolic DPPH solution. The mixture was vortexed vigorously and allowed to stand at room temperature for $30 \mathrm{~min}$ in the dark. Absorbance of the mixture was measured using a UV-Vis spectrometer at $517 \mathrm{~nm}$, and the free radical scavenging activity was calculated using Eq. (1):

$$
\text { \% Inhibition }=\left[1-\frac{\left(\mathrm{A}_{\mathrm{a}}-\mathrm{A}_{\mathrm{b}}\right)}{\mathrm{A}_{\mathrm{c}}}\right] \times 100
$$

where $A_{a}$ is the absorbance of the sample mixed with DPPH solution, $A_{b}$ is absorbance of the sample solutions, $A_{c}$ is absorbance of the control solutions. All the experiments were carried out in triplicate and the mean of the obtained results were reported.

\section{ABTS Radical Scavenging Assay}

The ABTS radical scavenging assay was performed using the method already reported in the literatures with slight modification [27, 28]. ABTS radical cations were produced from the reaction of ABTS $(7 \mathrm{mM})$ and potassium persulfate $(2.45 \mathrm{mM})$ by incubating the mixture at room temperature in the dark for $24 \mathrm{~h}$. The $\mathrm{ABTS}^{+}$solution diluted with deionized water to an absorbance below 1.0, which measured by using a UV-Vis spectrometer at $734 \mathrm{~nm}$. The different concentrations of AuNPs $(75,150,225,300$, and $375 \mu \mathrm{M})$ were added into the diluted ABTS $^{+}$ solution. The mixture solution was vortexed vigorously and stood at room temperature for $10 \mathrm{~min}$ in the dark. The absorbance was monitored at $734 \mathrm{~nm}$, and inhibition of ABTS was calculated in the same way of DPPH assay.

\section{Results and Discussion}

\section{Visual Observation and UV-Vis Spectral Studies}

Figure 1 showed the UV-Vis spectra and visual observation of the synthesized AuNPs under the various concentrations of $\mathrm{HAuCl}_{4} \cdot 4 \mathrm{H}_{2} \mathrm{O}$ solution. The color of the mixture changed from colorless to pale pink and finally to purple with the reaction time due to the excitation of surface plasmon vibrations [29]. When the AuNPs are small enough, their color is pinkish red because of their strong absorption of green light [12]. With the growth or aggregation of AuNPs, the color of the AuNPs changed to wine red or purple, which strongly depends on the shape and size of the synthesized AuNPs. Our present study showed the color changed faster at the lower concentration of 

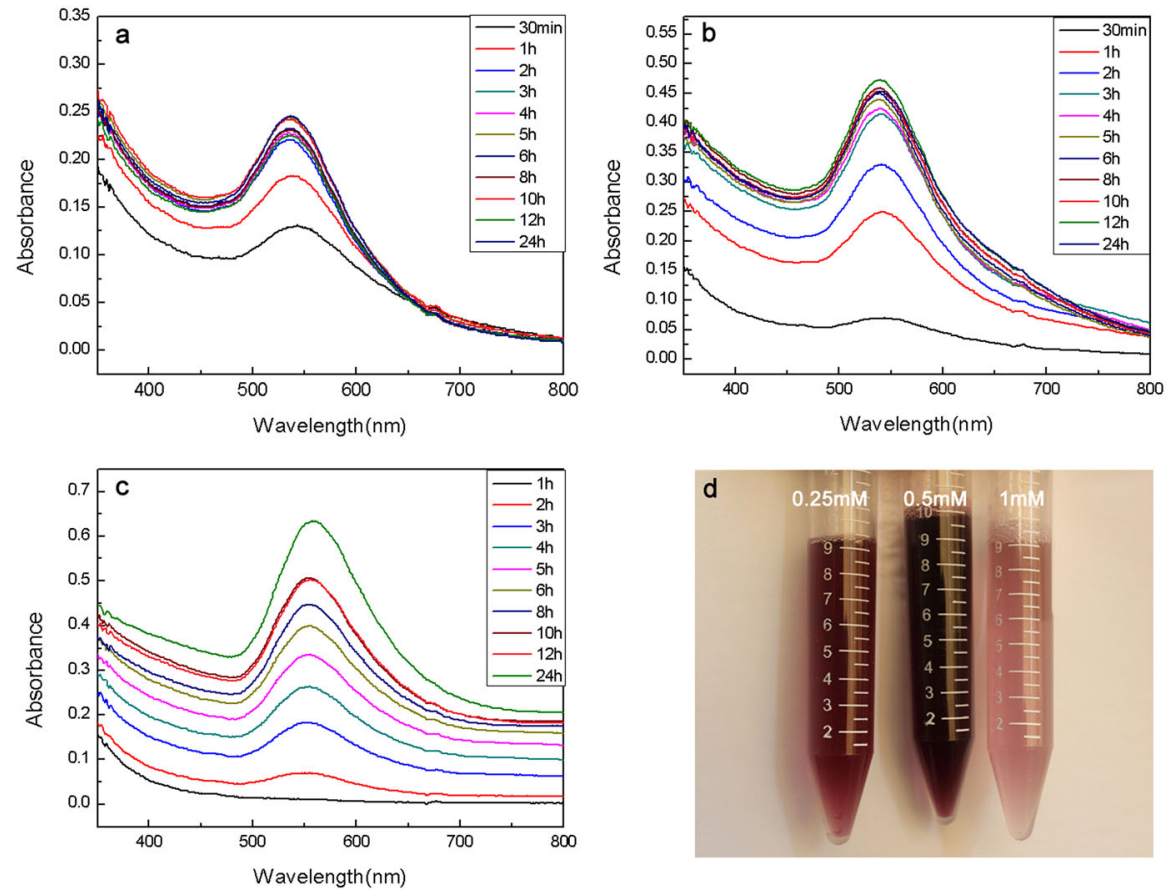

Fig. $1 \mathrm{UV}-$ Vis spectra of synthesized AuNPs under various $\mathrm{HAuCl}_{4} \cdot 4 \mathrm{H}_{2} \mathrm{O}$ concentrations a $0.25 \mathrm{mM}$, b $0.5 \mathrm{mM}$, c $1 \mathrm{mM}$, and d visual observation of synthesized AuNPs after $2 \mathrm{~h}$ reaction

$\mathrm{HAuCl}_{4} \cdot 4 \mathrm{H}_{2} \mathrm{O}$ than that at the higher. The abundant biomolecules gave sufficient reduction reagents for $\mathrm{Au}(\mathrm{III})$ ions at the lower concentration. The reduced $\mathrm{Au}(0)$ formed $\mathrm{Au}$ nuclei, and subsequently grown into the clusters by the continuous reduction of $\mathrm{Au}(\mathrm{III})$. At the same time, flavonoids, phenols, or terpenoids in the solution acted as the capping agents on the surface of the as prepared AuNPs to stabilize them through electrostatic repulsion. UV-Vis spectra also confirmed this phenomenon. It is clearly observed a distinct absorption peak appeared at $534 \mathrm{~nm}$ after 30 min reaction under the lower concentration of $\mathrm{HAuCl}_{4} \cdot 4 \mathrm{H}_{2} \mathrm{O}(0.25 \mathrm{mM})$, while a weak absorption peak appeared at the higher concentration of $\mathrm{HAuCl}_{4} \cdot 4 \mathrm{H}_{2} \mathrm{O}$. The color of the mixture at $0.5 \mathrm{mM}$ of $\mathrm{HAuCl}_{4} \cdot 4 \mathrm{H}_{2} \mathrm{O}$ was darker than that at the other concentrations (Fig. 1d) after $2 \mathrm{~h}$. The phenomenon was in accordance with the corresponding intensity of absorption peaks. However, the strongest SPR intensity was obtained at the higher concentration of $\mathrm{HAuCl}_{4} \cdot 4 \mathrm{H}_{2} \mathrm{O}(1 \mathrm{mM})$ after $24 \mathrm{~h}$, which indicated that more AuNPs were synthesized with sufficient $\mathrm{Au}$ (III) ions as the precursors. Furthermore, there was no evident shift of the SPR band during the process of AuNPs formation due to the favorable stability of the synthesized AuNPs by the biomolecules.

Temperature is one of the significant parameters that influence the reaction speed as well as the stability and the character of the synthesized AuNPs [30]. Figure 2 showed the UV-Vis spectra of the AuNPs at the various reaction temperatures. It was evidential that the AuNPs were rapidly synthesized at high temperature, which 

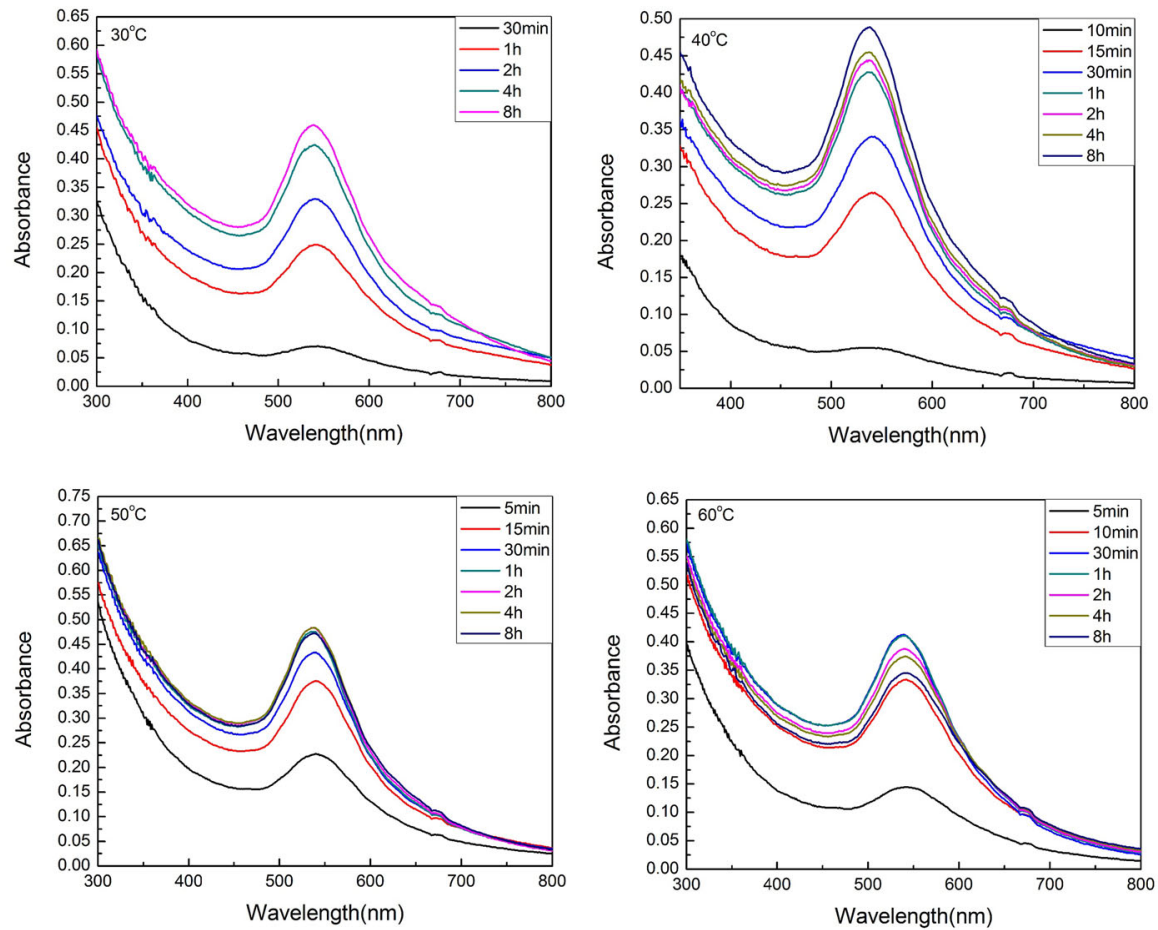

Fig. 2 UV-Vis spectra of synthesized AuNPs under various temperatures

was in agreement with our previous reports [31, 32]. The absorption peaks appeared only after $5 \mathrm{~min}$ at 50 or $60{ }^{\circ} \mathrm{C}$, and there was no change of the peak intensity after $1 \mathrm{~h}$ indicating the accomplishment of the reaction. In addition, the SPR band had little shift with the reaction time, which indicated that the favorable stability of the AuNPs could be obtained at different temperatures.

$\mathrm{pH}$ of the extract plays another crucial role in controlling size, size distribution, morphology and stability of the synthesized nanoparticles [33]. Our study showed that the intensity of absorption peaks elevated and the SPR band blue shifted with the increasing of $\mathrm{pH}$ (Fig. 3). The results indicated small sized AuNPs with

Fig. 3 UV-Vis spectra of synthesized AuNPs at the various $\mathrm{pH}$ values

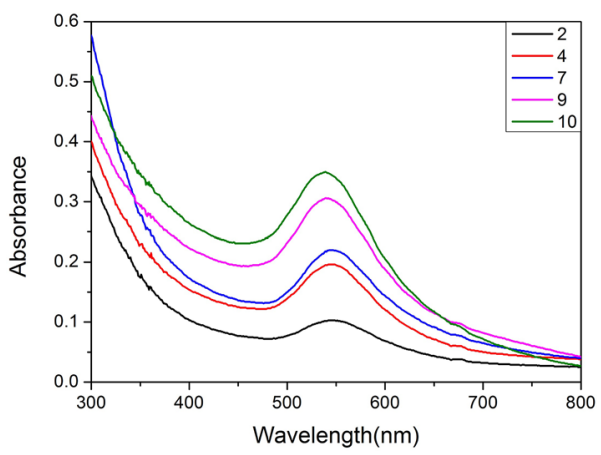


favorable dispersity were synthesized at the higher $\mathrm{pH}$, because the $\mathrm{Cl}^{-}$ions present in $\mathrm{AuCl}_{4}{ }^{-}$was substituted by $\mathrm{OH}^{-}$present in extract resulting in repulsion between the two negatively charged moieties of extract and gold ions, thereby reducing the further growth of nuclei to maintain them as small diameters [31, 34, 35].

\section{The Yield of the AuNPs}

The UV measurement can only demonstrate the process of biosynthesis reaction. It can not provide us the quantification information about the real yield of the formed NPs in the mixture. The yield of NPs is a significant factor to measure the potential of the plants in NPs synthesis and to provide the strong basis for industrial application. Several researchers optimized the physicochemical parameters to enhance the yield of biosynthesized NPs [36]. The increasing of UV absorbance value of the NPs colloid indicated the increasing of the NPs yield [37]. However, few researches focused on the quantity determination of the yield of synthesized NPs. Huang et al. [38] determined the yield of AgNPs synthesized with Cacumen Platycladi extract by deducting the residual $\mathrm{Ag}^{+}$ions in the supernatant after centrifugation from the total $\mathrm{Ag}^{+}$ions. Kang et al. [39] used the iodometry method to determine the yield of the AuNPs after separating the residual Au(III) ions and synthesized AuNPs using dialysis. In our present study, centrifugal ultrafiltration was used to separate Au(III) ions from AuNPs [25, 40]. The residual Au(III) ions in the supernatant were measured by ICP-MS, and the amount of AuNPs was calculated using Eq. (2):

$$
\mathrm{M}_{\mathrm{AuNPs}}=\mathrm{M}_{\mathrm{TAu}}-\mathrm{M}_{\mathrm{Au}(\mathrm{III})}
$$

where $M_{\text {AuNPs }}$ represented the mass of AuNPs, $M_{T A u}$ and $M_{A u(I I I)}$ were the initial mass of gold ions and the mass of the residual gold ions in the supernatant, respectively. The yields of AuNPs were $99.52 \%$ for $0.25 \mathrm{mM}$ of $\mathrm{Au}(\mathrm{III}), 99.87 \%$ for $0.5 \mathrm{mM}$, and $99.67 \%$ for $1.0 \mathrm{mM}$ after $1 \mathrm{~h}$ reaction at $60{ }^{\circ} \mathrm{C}$ (Fig. 4). Such high yields of AuNPs indicated that there are plenitudinous biomolecules in the $C$. aristatum $L$. stem extract that cause the sufficient reduction of $\mathrm{Au}(\mathrm{III})$ ions. The quantification results also demonstrated that this biosynthesis method was rapid and efficient.

Fig. 4 The yield of the synthesized AuNPs under various $\mathrm{HAuCl}_{4} \cdot 4 \mathrm{H}_{2} \mathrm{O}$ concentrations

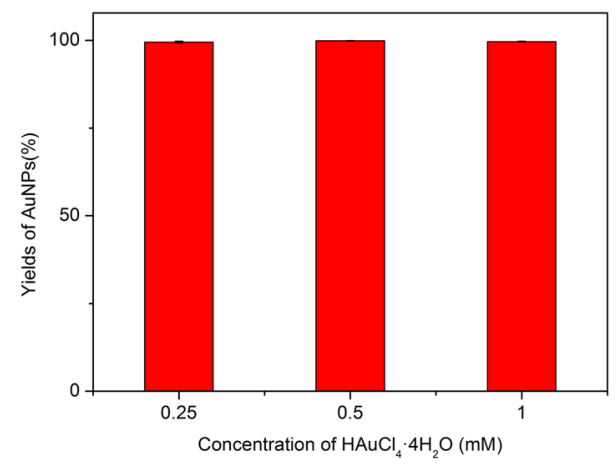




\section{TEM and EDS Analysis}

TEM images (Fig. 5a) showed the synthesized AuNPs were well dispersed, and their shapes were predominantly spherical with size range from 7 to $19 \mathrm{~nm}$. In addition, truncated triangular as well as small amounts of pentagonal, hexagonal, rod-like and other irregular shapes were also presented. The high resolution (HR) TEM images (Fig. 5b-f) clearly showed the different morphologies of the
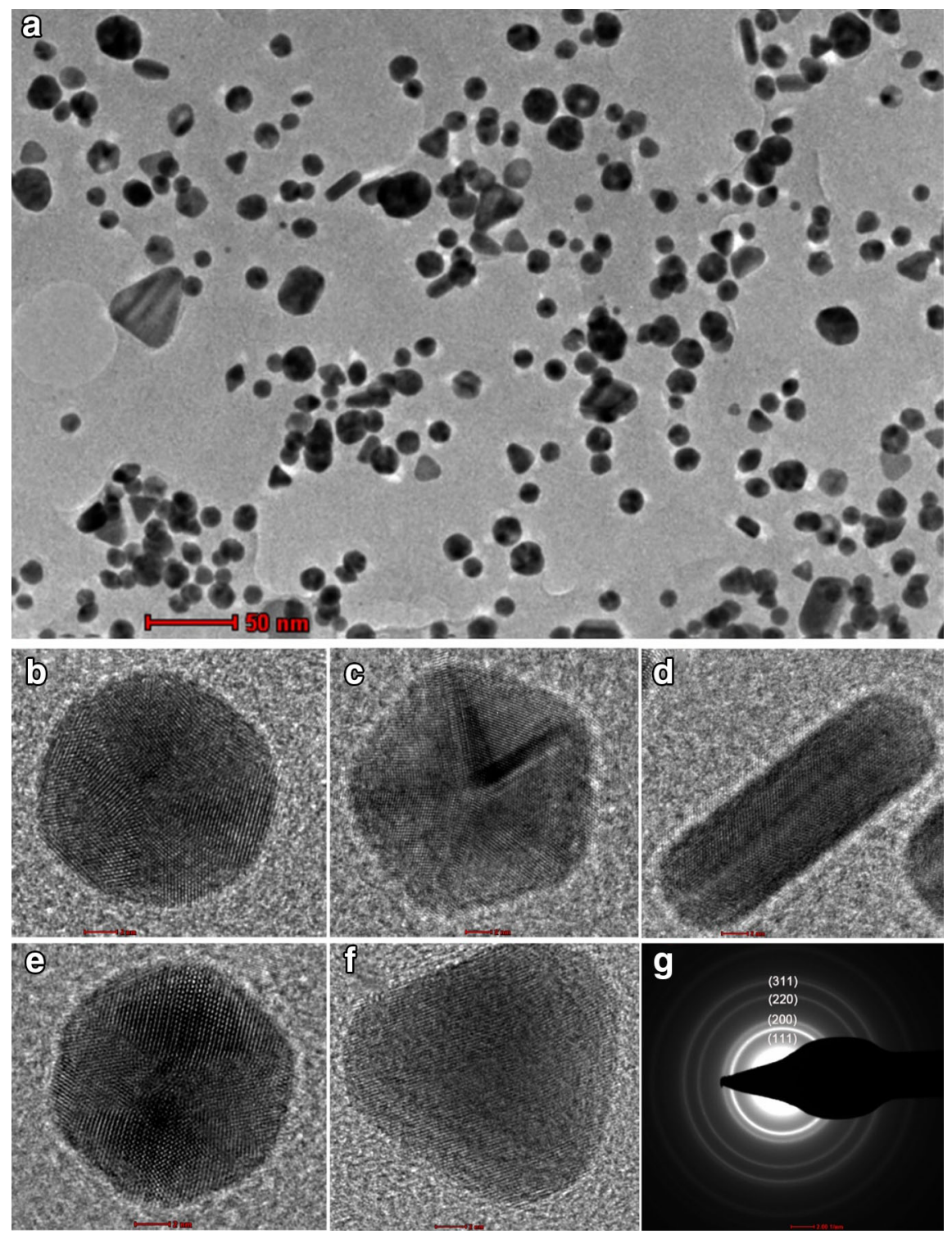

Fig. 5 a TEM images, b-f HRTEM images of AuNPs with different morphology, and g SAED pattern 


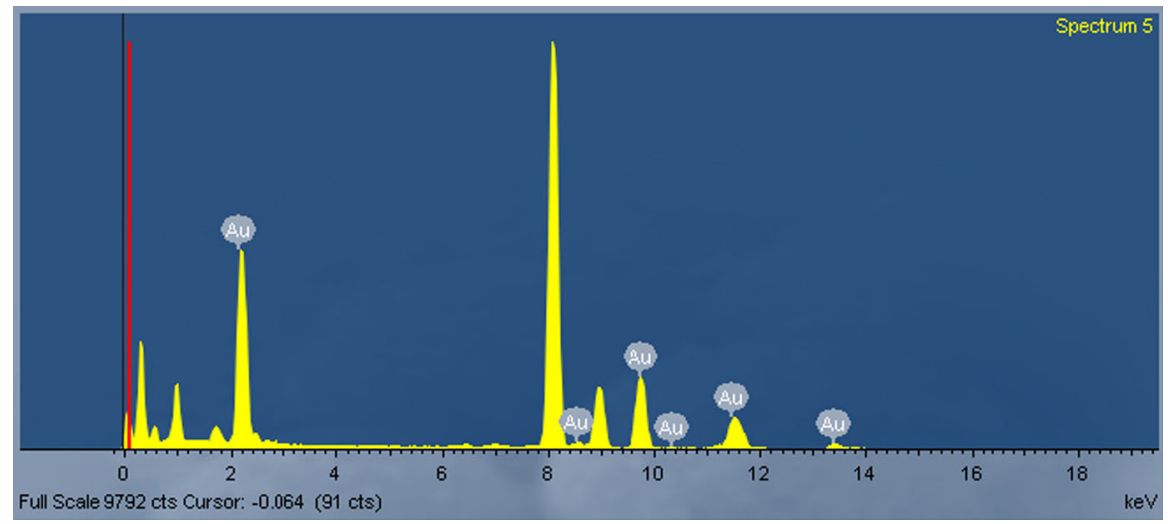

Fig. 6 EDS analysis of synthesized AuNPs

synthesized AuNPs. It is well known that the morphology of nanoparticles was strongly influenced by the biomolecules [41, 42]. C. aristatum L. stem extract contain amounts of bioactive compounds like reducing sugar, polyphenols. The cooperation of these various biomolecules gave rise to the formation of AuNPs with different morphologies. Some researches revealed that reducing sugar could mediate the formation of spherical AuNPs and prevent the nanoparticles from agglomeration $[41,42]$. Polyphenols were involved in the synthesis of gold nanoplates. Jiang et al. [42] demonstrated the adjacent phenolic hydroxyls of polyphenols could chelate with $\mathrm{Au}$ (III) to form a chelate complex. Then the adjacent phenolic hydroxyls are inductively oxidized to the corresponding carbonyls and ketones which were purposefully bound on the $\mathrm{Au}\{111\}$ plane to lead the nanoplate emerged. In addition, the coalescence of smaller particles also results in the formation of AuNPs with different morphology [10]. The crystalline nature of the synthesized AuNPs was confirmed by selected area electron diffraction (SAED) patterns (Fig. $5 \mathrm{~g}$ ) with bright circular rings corresponding to (111), (200), (220) and (311) planes, which corroborated well with the XRD patterns. From the EDS analysis (Fig. 6), the sharp peak was observed at $2.2 \mathrm{keV}$, which is typical for the absorption of metallic gold [43]. The peaks at 8.5, 9.7, 10.3, 11.4 and $13.4 \mathrm{keV}$ also confirmed the existence of the elemental gold.

\section{Zeta Potential Measurement}

AuNPs carried stable negative charges with a zeta $(\zeta)$ potential of $-22.9 \pm 5.56 \mathrm{mV}$, because the capping molecules like flavonoids and terpenoids were present on the surface of AuNPs, which were mainly comprised of negatively charged groups and responsible for the stability of the nanoparticles.

\section{XRD Analysis}

XRD analysis was used to confirm the crystal structure of the synthesized nanoparticles (Fig. 7). The diffraction peaks were located at $2 \theta=38.188^{\circ}, 44.552^{\circ}$, 
Fig. 7 XRD pattern of the synthesized AuNPs using $C$. aristatum $L$. stem extract

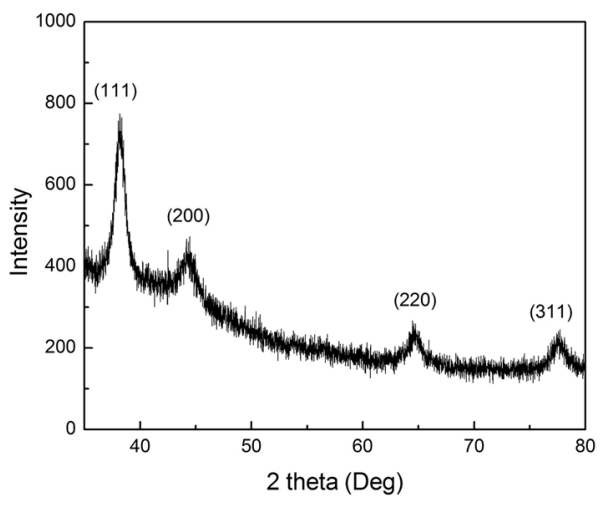

$64.589^{\circ}$, and $77.584^{\circ}$, which were corresponded to the (111), (200), (220), and (311) planes of the faced-centered cubic (fcc) lattice of gold. The patterns were matched well with JCPDS file No. 04-0784 [43, 44]. The mean size of the AuNPs was calculated using Debye-Scherrer's equation by determining the width of the (111) planes and found to be $\sim 16 \mathrm{~nm}$, which was in accordance with the result of TEM analysis.

\section{FTIR Analysis}

FTIR has emerged as the useful instrument to find the possible functional groups in synthesis of AuNPs. Figure 8 showed the FTIR spectrum of $C$. aristatum L. stem and the synthesized AuNPs. There was no significant shift between two spectra, which indicated that some functional groups from flavonoids, phenolic acids and terpenoids in $C$. aristatum $L$. stem were involved in the formation of AuNPs. The peak at $3420 \mathrm{~cm}^{-1}$ is corresponding to the stretching vibration for $\mathrm{O}-\mathrm{H}$ of phenols and carboxylic acids [45]. The peak at $2912 \mathrm{~cm}^{-1}$ is assigned to the symmetric and asymmetric $\mathrm{C}-\mathrm{H}$ stretching vibration. The peak at $1630 \mathrm{~cm}^{-1}$ corresponds to the amide I band of proteins released from the stem or the $\mathrm{C}=\mathrm{O}$ bond of the carbonyl

Fig. 8 FTIR spectra of $C$. aristatum L. stem and AuNPs

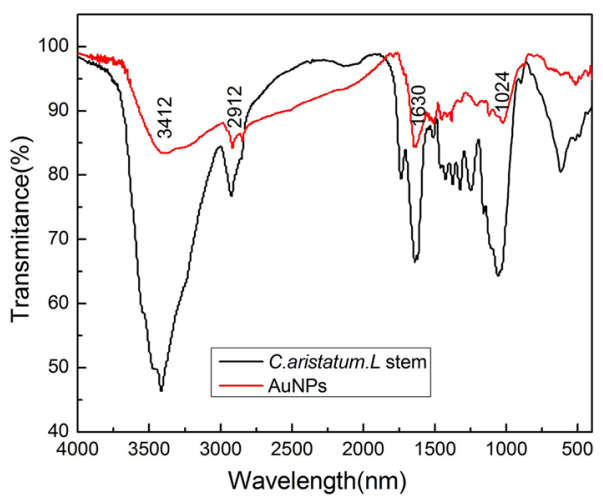


group [46]. The absorption peak at $1024 \mathrm{~cm}^{-1}$ may be assigned to $\mathrm{C}-\mathrm{O}-\mathrm{H}$ stretching which is typical for polysaccharides [47].

\section{Catalytic Activity}

4-Nitrophenol (4-NP) as a harmful and hazardous contaminant is typically found in industrial product and agricultural waste water. Usually hydrogenation of 4-NP to the 4-aminophenol (4-AP) is achieved in the presence of metal nanoparticles which act as catalyst and facilitate electron relay from the donor $\mathrm{BH}_{4}{ }^{-}$to the acceptor 4-NP to overcome the kinetic barrier [48]. In this study, the catalytic activity of the synthesized AuNPs was studied by degradation of 4-NP in the presence of $\mathrm{NaBH}_{4}$ at room temperature. The process of degradation was monitored by $\mathrm{UV}-\mathrm{V}$ is spectra. The 4-NP showed the absorption peak at $317 \mathrm{~nm}$, which red shift at $400 \mathrm{~nm}$ in the presence of fresh $\mathrm{NaBH}_{4}$ due to the formation of 4-nitrophenolate ion [48]. After the addition of AuNPs, the intensity of absorption peaks at $400 \mathrm{~nm}$ decreased and a new peak appeared at $\sim 310 \mathrm{~nm}$ due to the formation of 4-AP (Fig. 9a) [49]. Meanwhile, the color of the mixture faded with the reaction time. It consumed $30 \mathrm{~min}$ to complete the reaction, which was slow compared with our previous study [31, 32]. On the one hand, $40 \mu \mathrm{L}$ of $0.5 \mathrm{mM}$ AuNPs was used in the present study, but $40 \mu \mathrm{L}$ of $1 \mathrm{mM}$ AuNPs was added in our previous study. The higher concentration of catalyst could accelerate the reaction rate. On the other hand, many researches revealed the spherical AuNPs showed the higher catalytic activity than other shapes [50, 51]. Thus, the present AuNPs was consisted with the definite proportion of nanoplates, which could decrease the catalytic activity comparing with the pure spherical AuNPs. The concentration of borohydride ion can be considered as a constant due to the excessive addition of $\mathrm{NaBH}_{4}$. Hence, the reaction rate $(\mathrm{Ka})$ of reduction is assumed to be only dependent on the concentration of 4-NP and follow the first order kinetics. The rate equation was simplified and written as - Kat $=\ln \left(\mathrm{C}_{\mathrm{t}} / \mathrm{C}_{0}\right)=\ln \left(\mathrm{A}_{\mathrm{t}} / \mathrm{A}_{0}\right)$. Where $\mathrm{C}_{\mathrm{t}}$ and $\mathrm{A}_{\mathrm{t}}$ represent the concentration and absorption of the 4-nitrophenolate ion at any time $t$, while $\mathrm{C}_{0}$ and $\mathrm{A}_{0}$ are initial concentration and absorption of the reactant. Figure $9 \mathrm{~b}$ showed the linear
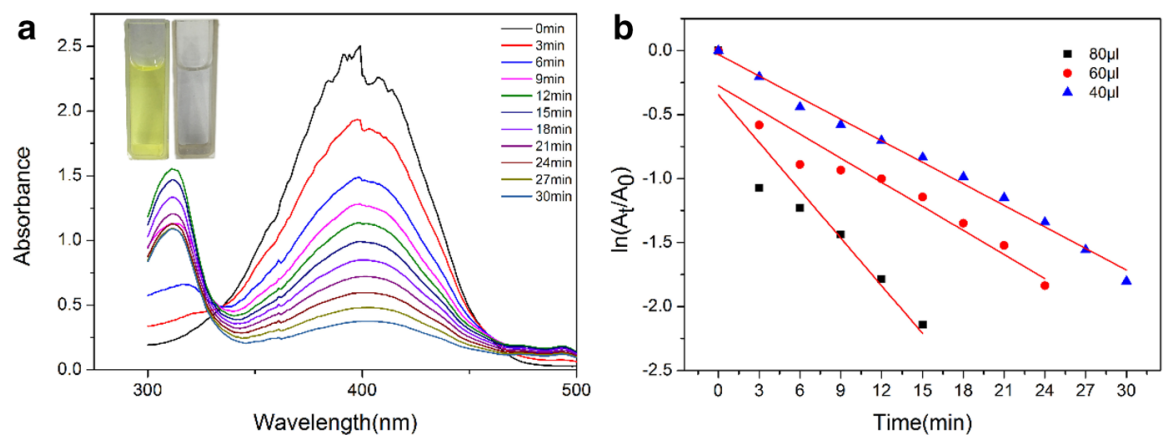

Fig. 9 a UV-Vis spectra of the degradation of 4-NP by synthesized AuNPs. The inset shows the color change of the reactant. b The logarithm of the ratio between its final concentration and the initial concentration of 4-NP versus corresponding time (min) 
relationship of $\ln \left(\mathrm{A}_{\mathrm{t}} / \mathrm{A}_{0}\right)$ versus corresponding reaction time. It was observed that the rate constant was increased with the increasing of AuNPs volume. The results demonstrated that the synthesized AuNPs showed the strong catalytic activity in the degradation of 4-NP.

\section{Antioxidant Activity of AuNPs}

\section{DPPH Radical Scavenging Activity}

It is well documented that flavonoids and polyphenols have ability to donate the electrons or hydrogens, which in turn reduce DPPH radicals [52]. Figure 10a showed the percent of inhibition of DPPH radical scavenging activity. Five different concentrations of AuNPs were tested and the inhibition efficiencies were found to increase in a dose-dependent manner. With the increasing of AuNPs concentration from 75 to $375 \mu \mathrm{M}$, the inhibition efficiency increased from 5.33 to $86.66 \%$, respectively. There were massive bioactive molecules in the C. aristatum $L$. stem extract and they coated on the surface of the synthesized AuNPs through electrostatic attraction, which improved the DPPH radical scavenging efficiency of the synthesized AuNPs [53].

\section{ABTS Radical Scavenging Activity}

The evaluation of the antioxidant behavior of biosynthesized nanoparticles is useful to establish their potential applications in the therapy of many diseases caused by inflammation and oxidative stress [54]. In our present study, the AuNPs exhibited the potential for scavenging $\mathrm{ABTS}^{+}$radical by surface reaction phenomenon in a dose dependent manner (Fig. 10b). With the enhancing of AuNPs concentration, the scavenging efficiency was enhanced from 24.02 to $89.95 \%$. Hence, the synthesized AuNPs using the $C$. aristatum $L$. stem extract demonstrated the favourable antioxidant activity.
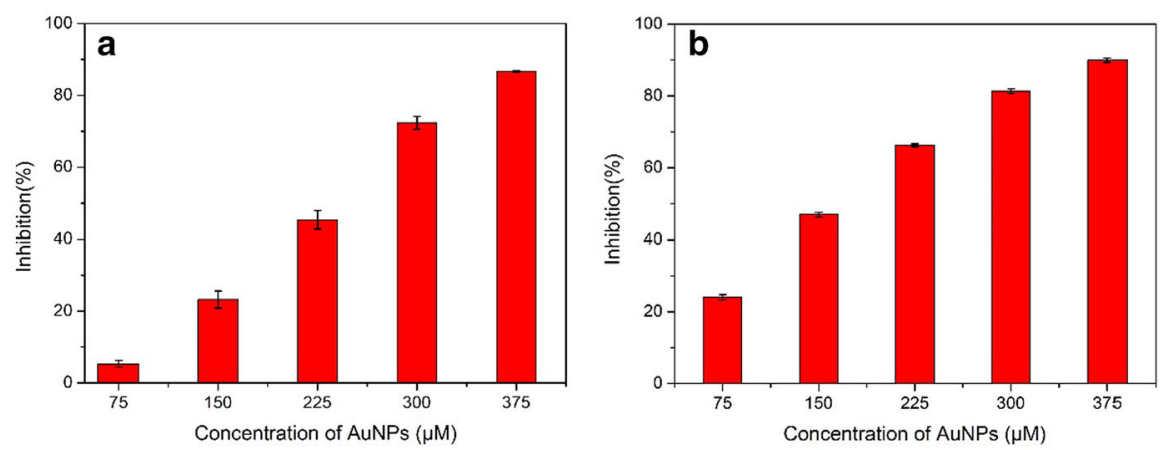

Fig. 10 a DPPH free radical scavenging activity, b ABTS free radical scavenging activity 


\section{Conclusion}

Our study proposed a cost-effective and rapid approach to synthesize AuNPs using Chenopodium aristatum $L$. stem extract. Chenopodium aristatum $L$. is a common obnoxious weed which has the large biomass and it can be a natural nano-factory for the preparation of AuNPs. The influences of the parameters including $\mathrm{HAuCl}_{4} \cdot 4 \mathrm{H}_{2} \mathrm{O}$ concentration, extract $\mathrm{pH}$ and reaction temperature on the synthesis rate and AuNPs morphology were also investigated and optimized. The yields of the reaction were quantified. ICP-MS measurement showed more than $99 \% \mathrm{Au}(\mathrm{III})$ ions were converted to AuNPs in $1 \mathrm{~h}$. The synthesized AuNPs were predominantly spherical, and small amounts of AuNPs in the other shapes were also found. XRD and SEDA patterns demonstrated that the AuNPs were in the face-centered cubic (fcc) structure. Zeta potential of the synthesized AuNPs was -22.9 $\pm 5.56 \mathrm{mV}$. The synthesized AuNPs showed the good catalytic activity and antioxidant activity, which indicated their potential applications in the future.

Acknowledgements This work was kindly co-funded by the National Natural Science Foundation of China (21620102008) and the Fundamental Research Funds for the Central Universities (2017ZZD07).

\section{References}

1. P. Puvanakrishnan, J. Park, D. Chatterjee, S. Krishnan, and J. W. Tunnell (2012). Int J Nanomed. 7, 1251.

2. D. R. Bhumkar, H. M. Joshi, M. Sastry, and V. B. Pokharkar (2007). Pharm. Res. 24, 1415.

3. E. Torres-Chavolla, R. J. Ranasinghe, and E. C. Alocilja (2010). IEEE T Nanotechnol. 9, 533.

4. B. S. Kim and J. Y. Song, in J. F. Shaw, C. T. Hou (eds) Biocatalysis and biomolecular engineering (2010). p. 447.

5. A. Timoszyk, J. Niedbach, P. Śliżewska, A. Mirończyk, and J. J. Kozioł (2015). Jokull. 65, 71.

6. T. S. Dhas, V. G. Kumar, V. Karthick, K. Vasanth, G. Singaravelu, and K. Govindaraju (2016). Enzym. Microb. Technol. 95, 100.

7. W. Shen, Y. Qu, X. Pei, X. Zhang, Q. Ma, Z. Zhang, S. Li, and J. Zhou (2016). Biotechnol. Lett. 38, 1503.

8. X. Zhang, Y. Qu, W. Shen, J. Wang, H. Li, Z. Zhang, S. Li, and J. Zhou (2016). Colloid Surf. A. 497, 280.

9. S. Iravani (2011). Green Chem. 13, 2638.

10. P. P. Gan and S. F. Y. Li (2012). Rev. Environ. Sci. Bio. 11, 169.

11. M. Ghaedi, S. Heidarpour, S. N. Kokhdan, R. Sahraie, A. Daneshfar, and B. Brazesh (2012). Powder Technol. 228, 18.

12. B. S. Srinath and R. V. Rai (2015). J. Clust. Sci. 26, 1483.

13. R. Mata, A. Bhaskaran, and S. R. Sadras (2015). Particuology 24, 78.

14. V. Vilas, D. Philip, and J. Mathew (2016). J. Mol. Liq. 221, 179.

15. G. Rajakumar, T. Gomathi, A. Abdul Rahuman, M. Thiruvengadam, G. Mydhili, S. H. Kim, T. J. Lee, and I. M. Chung (2016). Appl. Sci. 6, 222.

16. S. U. Ganaie, T. Abbasi, and S. A. Abbasi (2016). Environ. Prog. Sustain Energy. 35, 20.

17. A. A. Gohara and M. M. A. Elmazar (1997). Phytother. Res. 11, 564.

18. R. Y. Nsimba, H. Kikuzaki, and Y. Konishi (2008). Food Chem. 106, 760.

19. R. C. Ritva, H. Jarkkok, P. Juhamatti, and M. Pirjoh (2010). Food Chem. 120, 128.

20. A. I. Lukman, B. Gong, C. E. Marjo, U. Roessner, and A. T. Harris (2011). J. Colloid Interface Sci. 353, 433.

21. N. Ahmad, S. Sharma, M. K. Alam, V. N. Singh, S. F. Shamsi, B. R. Mehta, and A. Fatma (2010). Colloid Surf. B 81, 81 .

22. B. Kumar, K. Smita, and L. Cumbal (2016). J. Sol Gel Sci. Technol. 78, 285. 
23. Z. Q. Tan, J. F. Liu, X. R. Guo, Y. G. Yin, S. K. Byeon, M. H. Moon, and G. B. Jiang (2015). Anal Chem. 87, 8441.

24. X. X. Zhou, R. Liu, and J. F. Liu (2014). Environ. Sci. Technol. 48, 14516.

25. S. Yu, Y. Yin, J. Chao, M. Shen, and J. Liu (2014). Environ. Sci. Technol. 48, 403.

26. B. Kumar, K. Smita, L. Cumbal, and Y. Angulo (2015). J. Mol. Liq. 211, 476.

27. K. D. Lee, P. C. Nagajyothi, T. V. M. Sreekanth, and S. Park (2014). J. Ind. Eng. Chem. $26,67$.

28. B. Moldovan, O. Ghic, L. David, and C. Chisbora (2012). Rev. De. Chimie. 63, 463.

29. Y. Wang, X. He, K. Wang, X. Zhang, and W. Tan (2009). Colloid Surf. B. 73, 75.

30. S. Link and M. A. El-Sayed (1999). J. Phys. Chem. B. 103, 4212.

31. C. G. Yuan, C. Huo, S. Yu, and B. Gui (2017). Physica E 85, 19.

32. C. Yuan, C. Huo, B. Gui, and W. Cao (2016). Iet Nanobiotechnol. doi:10.1049/iet-nbt.2016.0183.

33. O. Velgosová, A. Mražíková, and R. Marcinčáková (2016). Mater. Lett. 180, 336.

34. M. Kumari, A. Mishra, S. Pandey, S. P. Singh, V. Chaudhry, M. K. R. Mudiam, S. Shukla, P. Kakkar, and C. S. Nautiyal (2016). Sci. Rep. UK 6, 27575.

35. K. S. Abhijith and M. S. Thakur (2012). Anal. Methods UK 4, 4250.

36. J. Bhaumik, N. S. Thakur, P. K. Aili, A. Ghanghoria, A. K. Mittal, and U. C. Banerjee (2015). ACS Biomater. Sci. Eng. 1, 382.

37. A. Bhargava, N. Jain, M. A. Khan, V. Pareek, R. V. Dilip, and J. Panwar (2016). J. Environ. Manag. 183, 22.

38. J. Huang, G. Zhan, B. Zheng, D. Sun, F. Lu, Y. Lin, H. Chen, Z. Zheng, Y. Zheng, and Q. Li (2011). Ind. Eng. Chem. Res. 50, 9095.

39. F. Kang, X. Qu, P. J. J. Alvarez, and D. Zhu (2017). Environ. Sci. Technol.. doi:10.1021/acs.est. $6 \mathrm{~b} 05930$.

40. Z. Zhang, X. Yang, M. Shen, Y. Yin, and J. Liu (2015). J. Environ. Sci. China 35, 62.

41. J. Huang, Q. Li, D. Sun, and Y. Lu (2007). Nanotechnology 18, 105104.

42. X. Jiang, N. He, H. Liu, J. Huang, T. odoom-Wubah, and Q. Li (2013). J. Nanopart. Res. $15,1741$.

43. N. Saha and S. D. Gupta (2016). J. Clust. Sci. 27, 1.

44. G. Balasubramani, R. Ramkumar, R. K. Raja, D. Aiswarya, C. Rajthilak, and P. Perumal (2016). J. Clust. Sci. 27, 1.

45. A. Alvarez-Ordóñez, J. Halisch, and M. Prieto (2010). Int. J. Food Microbiol. $142,97$.

46. N. Mude, A. Ingle, A. Gade, and M. Rai (2009). J. Plant Biochem. Biotechnol. $18,83$.

47. S. S. Shankar, A. Rai, B. Ankamwar, A. Singh, A. Ahmad, and M. Sastry (2004). Nat. Mater. 3, 482.

48. M. Nasrollahzadeh, S. M. Sajadi, and M. Khalaj (2014). RSC Adv. 4, 47313.

49. K. Kuroda, T. Ishida, and M. Haruta (2009). J. Mol. Catal. A Chem. 298, 7.

50. M. Kumari, A. Mishra, S. Pandey, S. Singh, V. Chaudhry, M. Mudiam, S. Shukla, P. Kakkar, and C. Nautiyal (2016). Sci. Rep. UK 6, 27575.

51. T. N. J. I. Edison, E. R. Baral, Y. R. Lee, and S. H. Kim (2016). J. Clust. Sci. 27, 285.

52. N. Muniyappan and N. S. Nagarajan (2014). Process Biochem. 49, 1054.

53. F. U. Khan, Y. Chen, N. U. Khan, Z. U. H. Khan, A. U. Khan, A. Ahmad, K. Tahir, L. Wang, M. R. Khan, and P. Wan (2016). J. Phototoch. Photobio. B. 164, 344.

54. B. Moldovan, L. David, M. Achim, S. Clichici, and G. A. Filip (2016). J. Mol. Liq. 221, 271. 